\title{
OCORRÊNCIA DE PERÍODOS SECOS NA REGIÃO DE RIO LARGO, ALAGOAS
}

\section{ANDRÉ LUIZ DE CARVALHO ${ }^{1}$, JOSÉ LEONALDO DE SOUZA², GUSTAVO BASTOS LYRA ${ }^{3}$, ANTHONY CARLOS SILVA PORFIRIO ${ }^{4}$}

\author{
${ }^{1}$ Universidade Federal de Pernambuco (UFPE), Departamento de Energia Nuclear, Recife, PE, Brasil \\ ${ }^{2}$ Universidade Federal de Alagoas (UFAL), Instituto de Ciências Atmosféricas, Maceió, AL, Brasil \\ ${ }^{3}$ Universidade Federal Rural do Rio de Janeiro (UFRRJ), Instituto de Florestas, Rio de Janeiro, RJ, Brasil \\ ${ }^{4}$ Instituto Nacional de Pesquisas Espaciais (INPE), Divisão de Satélites e Sistemas Ambientais, Cachoeira \\ Paulista, SP, Brasil
}

del.andre2@hotmail.com, leonaldojs@yahoo.com.br,gblyra@ufrrj.br, anthonycsp@gmail.com

Recebido Outubro de 2011 - Aceito Outubro de 2012

\begin{abstract}
RESUMO
A disponibilidade de água de uma região está diretamente relacionada a frequência e a magnitude dos eventos de chuva. Maiores ocorrências de dias caracterizados como seco podem gerar diversos prejuízos, principalmente, para a agricultura. Assim, o objetivo deste trabalho foi determinar a probabilidade das ocorrências de períodos secos em Rio Largo, Alagoas, e relacioná-la a fenômenos de escala climática, tais como, El Niño Oscilação Sul (El Niño e La Niña). Os períodos secos foram avaliados em três diferentes intervalos de tempo com sequência de dias secos $(\geq 5$ dias, $\geq 7$ dias e $\geq 10$ dias). $\mathrm{O}$ dia foi definido seco com base em diferentes limites de precipitação $(\leq 0,1,2,3,4$ e $5 \mathrm{~mm}$ ). O fim de cada período seco foi determinado pela ocorrência de um dia chuvoso. As análises foram realizadas com a utilização do aplicativo Instat Climatic. O intervalo de 5 dias mostrou maior ocorrência na estação seca e chuvosa. Na estação chuvosa (abril a agosto), esse período seco apresentou probabilidade elevada de ocorrência (5 a 85\%). Enquanto, a ocorrência de períodos secos de 7 dias (0 a 45\%) e 10 dias (0 a 5\%) foi inferior. Notou-se forte influência de eventos de ENOS (El Niño Oscilação Sul) nas ocorrências dos períodos secos da região. Em anos de El Niño, as ocorrências de períodos secos foram intensificadas ( 10 a 60\%), e reduzidas em anos de eventos de La Niña atingindo (0 a $45 \%)$.
\end{abstract}

Palavras-chave: precipitação pluvial, dias secos, ENOS

ABSTRACT: OCURRENCE OF DRY PERIODS IN RIO LARGO, ALAGOAS, BRAZIL: Water availability in a region is directly related to intensity and frequency of rain occurrence. Major occurrences of days with low amounts of precipitation can produce many losses, especially for agriculture. Thus, the objective of this study was to determine the probability of occurrence of dry periods in Rio Largo, Alagoas, Brazil, and to relate it to climate scale phenomena such as El Niño - Southern Oscillation (El Niño and La Niña). The dry periods were evaluated for three different time intervals with sequence of dry days ( $\geq 5$ days, $\geq 7$ days and $\geq 10$ days). The sequence of days was defined as dry based on different thresholds of precipitation $(\leq 0,1,2,3,4$ and $5 \mathrm{~mm})$. The end of each dry period was determined by the occurrence of a rainy day. Analyses were performed using the Instat Climatic application. The 5 days interval was the most frequent in the dry and rainy seasons. In the rainy season (April-August), this dry period had higher frequency of occurrence of $5-85 \%$. While the occurrence of dry periods of 7 days ( 0 to $45 \%)$ and 10 days ( 0 to $5 \%$ ) was lower. It was noted the strong influence of ENSO events (El Niño Southern Oscillation) in the occurrence of dry periods. In El Niño years, the occurrences were enhanced (10-60\%), and reduced in years of La Niña events $(0-45 \%)$.

Keywords: rainfall, dry days, ENSO. 


\section{INTRODUÇÃO}

Nos Trópicos, a precipitação pluvial é um dos principais elementos climáticos, além de ser caracterizada por grande variação espacial e temporal na sua ocorrência (Kousky, 1980). No Nordeste do Brasil (NEB), sua variabilidade interanual e intrasazonal é, principalmente, influenciada por mecanismos de escala global como eventos de ENOS (El Niño e La Niña), que são considerados como a principal causa das variações no clima em diversas regiões do globo (Kousky, 1979). A ocorrência de extremos de precipitação está relacionada a enchentes (fortes chuvas) e secas prolongadas (Marengo et al., 2010). Em determinadas regiões, períodos sem ocorrências de precipitação tornam a água um recurso de baixa disponibilidade e até escasso e assim, contribuem para a migração da população para outras regiões.

A ausência ou quantidade de chuva inferior à necessidade de certa atividade humana (e.g. agricultura), em escala temporal, determina a formação de períodos secos. $\mathrm{O}$ inverso de período seco, ou seja, a sequência diária com quantidade superior a um limite adotado de precipitação é caracterizado como período chuvoso (Barron et al., 2003). Esse valor limite de precipitação depende das condições do clima e do solo da região (Vasconcellos et al., 2003), tais como as características físicas do solo, profundidade efetiva do sistema radicular e a necessidade hídrica das culturas (evapotranspiração). Na literatura, diversos são os valores adotados de precipitação diária como indicativo de períodos secos, tais como: $0,1 \mathrm{~mm}$ (Keller Filho et al., 2006), 0,85 mm (Barron et al., 2003), 1 mm (Santos et al., 2009; Arruda e Pinto, 1980) e 5 mm (Assad et al, 1993; Fietz et al., 1998; Viana et al., 2002). Porém, esses valores dependem da atividade e tipo de manejo ambiental em desenvolvimento. Alguns autores (Andrade Junior et al., 2001; Viana et al., 2002) definiram o dia como seco, baseando-se na ocorrência de déficit hídrico, ou seja, quando a precipitação foi igual a zero ou inferior a evapotranspiração de referência. Além desses, Vasconcellos et al. (2003) definiram o dia como seco, quando o armazenamento de água no solo, de acordo com o balanço hídrico, foi igual ou inferior a certo valor crítico, condicionado pela demanda atmosférica.

As ocorrências de períodos secos são fortemente influenciadas pelas condições climáticas da região. Farmer e Wigley (1985) comentam que as causas mais prováveis das ocorrências de períodos secos são: a temperatura da superfície do mar (TSM), as condições da superfície do continente (albedo e umidade) e a umidade atmosférica. Nos Trópicos, as ocorrências de períodos secos estão associadas, principalmente, ao posicionamento da Zona de Convergência Intertropical - ZCIT (Nieuwolt, 1989). Os períodos secos também estão associados aos eventos de ENOS - El Niño / Oscilação Sul, sendo esse diretamente relacionado a variações da temperatura do Oceano Pacífico Tropical Sul (Assad et al., 1993). Seu impacto está diretamente relacionado à baixa disponibilidade de água no solo, que dificulta, principalmente, o desenvolvimento da agricultura (provocando perdas na produtividade das plantas) e a criação de animais, que resulta em consequências negativas à economia da região. Com isso, a ocorrência de períodos secos pode provocar escassez de alimentos, má nutrição da população, doenças não verificadas, redução dos rebanhos de gado e perdas de vidas humana (Morse, 1987). As informações sobre períodos secos são essenciais para a agricultura, principalmente para avaliar o déficit de água para as culturas. Essas informações também são úteis para definir se uma cultura ou variedade é apta a ser cultivada em determinada região. Adequações de fenologia de uma cultura e o regime de precipitação podem ser feitos com as informações sobre períodos secos, antes que experimentos de campo sejam realizados (Sivakumar, 1992).

De maneira geral, espera-se que nos cenários de mudanças climáticas, em um futuro próximo, ocorram mais frequentemente eventos extremos, como por exemplo, estiagens mais frequentes (Marengo et al., 2010). Assim, esse trabalho objetivou determinar a probabilidade das ocorrências de períodos secos em Rio Largo, Alagoas, relacionando-a a fenômenos de escala climática, tais como El Niño Oscilação Sul (El Niño e La Niña).

\section{MATERIAL E MÉTODOS}

O estudo foi realizado com dados observados de precipitação pluvial diária entre 1973 e 2008 na região de Rio Largo ( $9^{\circ} 28^{\prime} \mathrm{S}, 35^{\circ} 49^{\prime} \mathrm{O}, 127 \mathrm{~m}$ ), obtidos em pluviômetro convencional, instalado na estação agrometeorológica no Centro de Ciências Agrárias (CECA) da Universidade Federal de Alagoas (UFAL).

O município de Rio Largo está localizado na Região Metropolitana de Maceió e região dos tabuleiros costeiros de Alagoas. O clima dessa região, segundo a classificação climática de Köppen, é tropical litorâneo úmido (As), com média anual da temperatura do ar mínima e máxima de 17,2 e $35,2^{\circ} \mathrm{C}$, respectivamente. A distribuição interanual da precipitação pluvial é bastante variável, apresentando média anual de $1.800 \mathrm{~mm}$, e associa-se à ocorrência de eventos de El Niño e La Niña. O período chuvoso inicia-se na primeira quinzena de abril e ocorre até a segunda quinzena de agosto. Nesse período, o total de chuva corresponde a $70 \%$ do total anual, com máxima ocorrência mensal em junho (301,1 mm). O período menos chuvoso inicia-se na primeira quinzena de outubro e ocorre até a primeira quinzena de fevereiro. O total de chuva nesse período é $16 \%$ do total anual, com mínima ocorrência mensal em dezembro (43,2 mm) (Souza et al., 2004; Cardim, 2003; Molion e Bernardo, 2002). 
$\mathrm{Na}$ definição de dia seco foram considerados seis diferentes valores de referência para a precipitação $(0,1,2,3$, 4 e $5 \mathrm{~mm}$ ). Dias com precipitação igual ou inferior a esse valor de referência foram considerados secos e dias com precipitação superior foram considerados chuvosos. Os períodos secos foram analisados em três diferentes intervalos de tempo ( $\geq 5$ dias, $\geq 7$ dias e $\geq 10$ dias), definidos através de análises das ocorrências de dias secos e chuvosos, sendo o fim de cada intervalo determinado pela ocorrência de um dia chuvoso. As análises foram realizadas com o auxílio do aplicativo Instat Climatic, que é uma ferramenta para análises estatísticas de eventos climáticos (Stern et al., 2005). A probabilidade de ocorrência de dias secos e chuvosos foi modelada pela utilização da distribuição gama (Buishand, 1977; Stern et al., 1982a; 1982b). A função densidade de probabilidade para a distribuição gama é dada por:

$$
\begin{aligned}
& f(x)=\frac{\lambda^{r}}{\Gamma(r)} x^{r-1} e^{-\lambda x} \quad \text { para } \mathrm{x} \geq 0 \\
& \mathrm{e}(x)=0 \quad \text { para } \mathrm{x}<0
\end{aligned}
$$

em que, os parâmetros da distribuição gama, que podem assumir qualquer valor positivo, são: $\lambda$, taxa média do processo; $r$, número específico de eventos que ocorrem até que a variável $\mathrm{X}$ (tamanho do segmento de tempo ou espaço) seja atingida e $\Gamma(\mathrm{r})$ é a função gama, definida por:

$$
\Gamma(r)=\int_{0}^{\infty} x^{r-l} e^{-x} d x \quad \text { para } \mathrm{r}>0 .
$$

A análise das características da estação chuvosa com os eventos de ENOS - El Niño Oscilação Sul foi realizada pela classificação dos anos; em anos de ocorrência de eventos de El Niño e anos de ocorrência de eventos de La Niña, para a série de dados de estudo, disponibilizada pelo Centro de Previsão de Tempo e Estudos Climáticos / Instituto Nacional de Pesquisas Espaciais (CPTEC/INPE) no site: http://enos.cptec.inpe.br/tab_elnino. shtml e http://enos.cptec.inpe.br/tab_lanina.shtml (Tabela 1).

\section{RESULTADOS E DISCUSSÕES}

A análise de eventos diários de períodos secos mostrou que as maiores probabilidades de ocorrência foram para períodos secos de cinco dias (Figura 1). Na estação seca (setembro - março) as ocorrências de períodos secos foram altas, independente do limite diário de precipitação adotado. Nessa estação, os períodos secos de cinco dias apresentaram probabilidade que variou de 60 a 100\% de chances de ocorrência, considerando todos os valores de precipitação de referência ( 0 a $5 \mathrm{~mm}$ ). Para os períodos de sete e dez dias, a probabilidade variou de 30 a $100 \%$ e 10 a $100 \%$, respectivamente. Azevedo e Leitão (1990) ao analisar as ocorrências dos períodos secos na região da Paraíba, constataram que, em média, 90\% das ocorrências são para períodos secos igual ou inferior a dez dias. Como esperado, as ocorrências de períodos secos entre setembro e março foram elevadas, devido a pouca ocorrência de chuva observada nesse período (Souza et al., 2004). Andrade et al. (2008) avaliaram o comportamento de períodos secos e chuvosos para Garanhuns/PE e concluíram que entre outubro e dezembro são esperados que ocorra o maior número de dias secos, com probabilidades de aproximadamente $88 \%$.

As ocorrências de períodos secos foram máximas entre os meses de novembro e janeiro (Figura 1). Para o período de cinco dias a probabilidade máxima variou de 90 a 100\% ( 0 a 5 $\mathrm{mm}$ ), enquanto que para sete e dez dias variou de 70 a $100 \% \mathrm{e}$ 40 a 100\%, respectivamente. A Tabela 2 mostra as ocorrências

Tabela 1 - Classificação e Intensidade do El Niño - Oscilação Sul no período de 1973 a 2008.

\begin{tabular}{ccc}
\hline Período & Classificação & Intensidade \\
\hline $1972-1973$ & El Niño & Forte \\
$1973-1976$ & La Niña & Forte \\
$1976-1977$ & El Niño & Fraco \\
$1977-1978$ & El Niño & Fraco \\
$1979-1980$ & El Niño & Fraco \\
$1982-1983$ & El Niño & Forte \\
$1983-1984$ & La Niña & Fraco \\
$1984-1985$ & La Niña & Fraco \\
$1986-1988$ & El Niño & Moderado \\
$1988-1989$ & La Niña & Forte \\
$1990-1993$ & El Niño & Forte \\
$1994-1995$ & El Niño & Moderado \\
$1995-1996$ & La Niña & Fraco \\
$1997-1998$ & El Niño & Forte \\
$1998-2001$ & La Niña & Moderado \\
$2002-2003$ & El Niño & Moderado \\
$2004-2007$ & El Niño & Fraco \\
2008 & La Niña & Forte \\
\hline
\end{tabular}


de períodos secos para o ano de 2008, com limite de precipitação de $1 \mathrm{~mm}$, valor mais utilizado na definição de dia seco. Nesse ano, o mês de novembro apresentou um período seco de 40 dias e apenas um dia chuvoso. Esse período seco começou no dia 15 de outubro e terminou no dia 23 de novembro. Dezembro também teve poucos dias chuvosos (três dias) e períodos secos longos: um com 14 dias, que teve início no dia 25 de novembro, e outro com 20 dias que teve toda a sua duração nesse mês.

Após o período entre setembro e março notou-se redução acentuada nas probabilidades de ocorrência de períodos secos. Isso ocorreu devido à proximidade da estação chuvosa, onde ocorre o máximo de chuva (Souza et al., 2004). Segundo Molion e Bernardo (2002), esse máximo de chuva estaria ligado à maior atividade de circulação de brisas que advecta bandas de nebulosidade para o continente e à ação de frentes frias, que se propagam ao longo da costa. Entre os meses de abril a
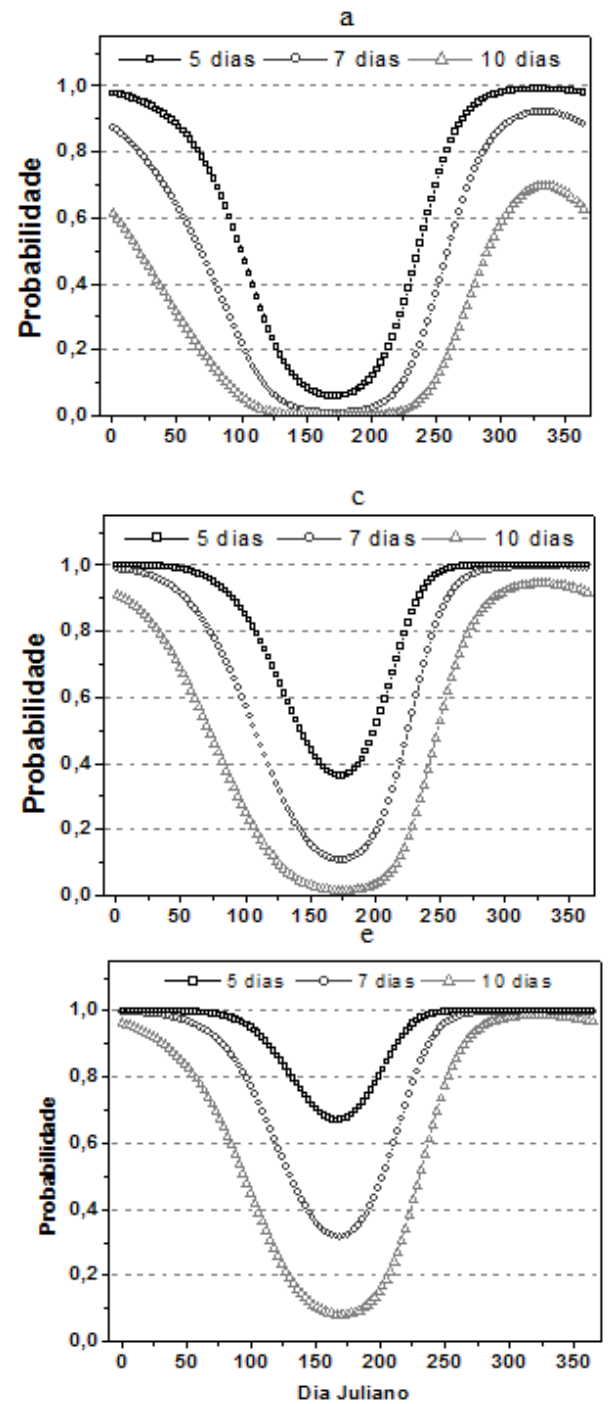

agosto observou-se que as ocorrências de períodos secos foram menores, principalmente para períodos secos de sete e dez dias (Figura 1). Para períodos secos de cinco dias as ocorrências variaram de 5 a $98 \%$. Para sete e dez dias a probabilidade variou de 0 a $80 \%$ e de 0 a $50 \%$, respectivamente.

As menores ocorrências de períodos secos ocorreram entre junho e julho. A probabilidade variou de 5 a $80 \%$, para períodos secos de cinco dias entre todos os valores de referência de precipitação utilizados. Para sete e dez dias a probabilidade variou de 0 a $48 \%$ e 0 a $18 \%$, respectivamente. Os meses de junho e julho são caracterizados por maiores ocorrências de dias chuvosos, e assim, a maior probabilidade de ocorrer períodos secos foram para períodos de dois dias (Carvalho, 2010). A média de dias chuvosos nesses meses para a Região foi de 14,7 e 14,9, respectivamente (Pereira et al., 2012). Essa baixa probabilidade de ocorrer um período seco mais longo significa
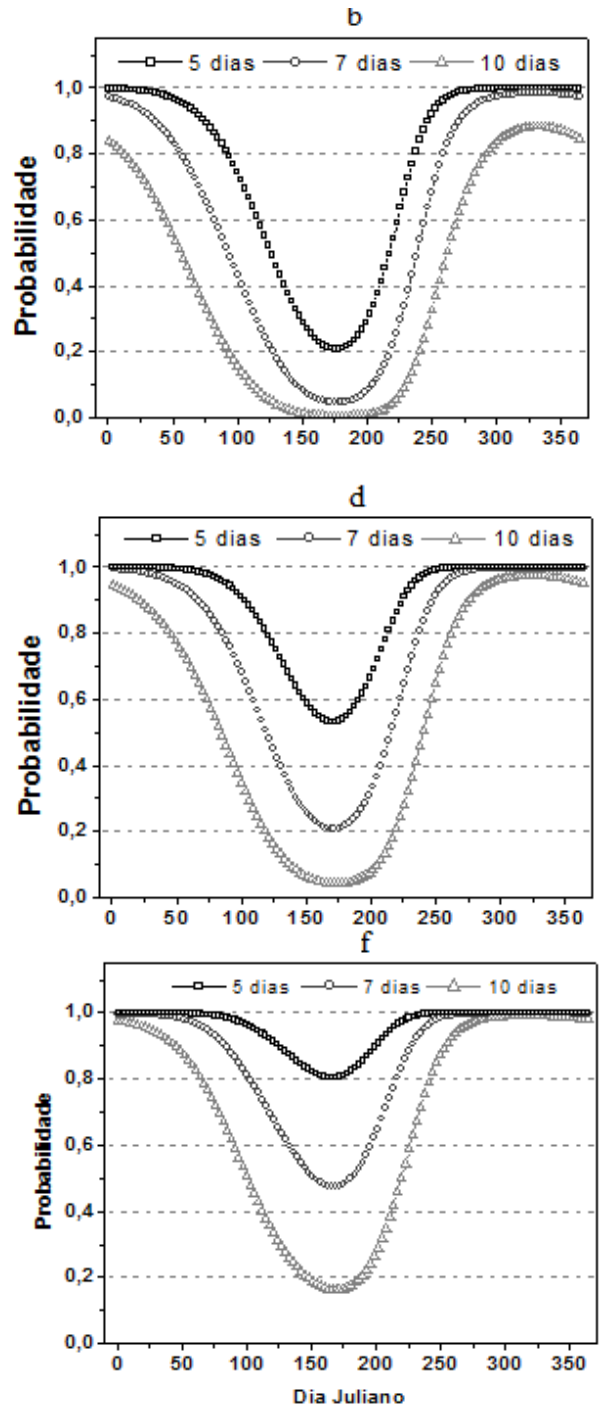

Figura 1 - Probabilidade média diária da ocorrência de períodos secos ( $\geq 5$ dias, $\geq 7$ dias, $\geq 10$ dias), no período de 1973 - 2008 , Rio Largo - AL, considerando dias sem precipitação (a), $1 \mathrm{~mm}$ (b), $2 \mathrm{~mm}$ (c), $3 \mathrm{~mm}$ (d), $4 \mathrm{~mm}$ (e) e $5 \mathrm{~mm}$ (f), como limites de precipitação pluvial de dias secos. 
Tabela 2 - Ocorrência sequencial de dias secos no ano de 2008 para o limite de $1 \mathrm{~mm}$ de precipitação para a região de Rio Largo, Alagoas.

\begin{tabular}{|c|c|c|c|c|c|c|c|c|c|c|c|c|}
\hline Dia & Jan & $\mathrm{Fev}$ & Mar & Abr & Mai & Jun & Jul & Ago & Set & Out & Nov & Dez \\
\hline 1 & 3 & 3 & 3 & 1 & 0 & 0 & 0 & 1 & 0 & 2 & 18 & 7 \\
\hline 2 & 4 & 4 & 0 & 0 & 1 & 0 & 0 & 0 & 0 & 3 & 19 & 8 \\
\hline 3 & 5 & 5 & 1 & 1 & 2 & 0 & 0 & 0 & 0 & 0 & 20 & 9 \\
\hline 4 & 6 & 6 & 2 & 2 & 0 & 0 & 0 & 0 & 0 & 1 & 21 & 10 \\
\hline 5 & 7 & 7 & 3 & 3 & 0 & 0 & 0 & 1 & 1 & 2 & 22 & 11 \\
\hline 6 & 8 & 8 & 4 & 0 & 1 & 0 & 0 & 0 & 2 & 3 & 23 & 12 \\
\hline 7 & 9 & 9 & 5 & 1 & 2 & 1 & 0 & 0 & 3 & 4 & 24 & 13 \\
\hline 8 & 10 & 10 & 0 & 0 & 0 & 0 & 0 & 0 & 4 & 0 & 25 & 14 \\
\hline 9 & 0 & 11 & 1 & 1 & 0 & 0 & 0 & 0 & 5 & 0 & 26 & 0 \\
\hline 10 & 1 & 12 & 2 & 2 & 0 & 0 & 0 & 1 & 0 & 0 & 27 & 0 \\
\hline 11 & 2 & 13 & 0 & 3 & 0 & 1 & 0 & 2 & 0 & 0 & 28 & 0 \\
\hline 12 & 3 & 14 & 1 & 4 & 0 & 2 & 0 & 0 & 1 & 1 & 29 & 1 \\
\hline 13 & 0 & 15 & 0 & 5 & 0 & 0 & 0 & 0 & 2 & 0 & 30 & 2 \\
\hline 14 & 0 & 16 & 1 & 6 & 1 & 0 & 1 & 0 & 3 & 0 & 31 & 3 \\
\hline 15 & 0 & 0 & 2 & 7 & 2 & 1 & 2 & 1 & 4 & 1 & 32 & 4 \\
\hline 16 & 1 & 0 & 0 & 8 & 0 & 0 & 0 & 0 & 5 & 2 & 33 & 5 \\
\hline 17 & 2 & 0 & 0 & 9 & 0 & 0 & 0 & 0 & 6 & 3 & 34 & 6 \\
\hline 18 & 3 & 1 & 0 & 0 & 0 & 0 & 0 & 0 & 7 & 4 & 35 & 7 \\
\hline 19 & 4 & 2 & 0 & 0 & 0 & 0 & 0 & 0 & 8 & 5 & 36 & 8 \\
\hline 20 & 5 & 0 & 0 & 0 & 1 & 0 & 0 & 0 & 9 & 6 & 37 & 9 \\
\hline 21 & 6 & 1 & 0 & 0 & 0 & 1 & 0 & 0 & 10 & 7 & 38 & 10 \\
\hline 22 & 7 & 2 & 0 & 0 & 0 & 0 & 0 & 0 & 11 & 8 & 39 & 11 \\
\hline 23 & 8 & 3 & 0 & 1 & 0 & 0 & 1 & 0 & 12 & 9 & 40 & 12 \\
\hline 24 & 0 & 4 & 0 & 2 & 0 & 0 & 0 & 0 & 13 & 10 & 0 & 13 \\
\hline 25 & 1 & 5 & 0 & 3 & 0 & 0 & 0 & 0 & 0 & 11 & 1 & 14 \\
\hline 26 & 2 & 6 & 0 & 4 & 0 & 1 & 0 & 0 & 1 & 12 & 2 & 15 \\
\hline 27 & 3 & 0 & 1 & 5 & 0 & 0 & 0 & 0 & 2 & 13 & 3 & 16 \\
\hline 28 & 0 & 1 & 2 & 0 & 0 & 0 & 0 & 0 & 3 & 14 & 4 & 17 \\
\hline 29 & 0 & 2 & 0 & 1 & 0 & 0 & 1 & 1 & 0 & 15 & 5 & 18 \\
\hline 30 & 1 & & 0 & 0 & 0 & 1 & 0 & 2 & 1 & 16 & 6 & 19 \\
\hline 31 & 2 & & 0 & & 0 & & 0 & 3 & & 17 & & 20 \\
\hline
\end{tabular}

que se espera quantidade satisfatória de água disponível no solo, o que favorece a produção agrícola da região.

A análise da distribuição da probabilidade de ocorrência de períodos secos para cada ano da série estudada mostrou que o período de cinco dias foi o mais observado (Figura 2). A probabilidade de ocorrência de períodos secos de cinco dias variou de aproximadamente 6 a $64 \%$, para 0 e $5 \mathrm{~mm}$ de precipitação como limite para dia seco, respectivamente. Para os períodos secos de sete e dez dias as probabilidades foram baixas com variação em torno de 2 a $55 \%$ e 0 a $45 \%$, respectivamente. Entre os anos de 1980 a 1983, observou-se aumento significativo nas ocorrências de períodos secos (de 10 a $60 \%$ ) entre todos os limites de precipitação adotados para dia seco. $\mathrm{O}$ aumento de períodos secos entre esses anos deve-se a ocorrências de eventos de El Niño, já que no ano de 1980 ocorreu um evento fraco de El Niño (Tabela 1) e nos anos seguintes foi registrado um evento forte de El Niño (1982 a 1983). Souza et al. (2004) mostraram que a quantidade média de precipitação pluvial, em anos de ocorrência de eventos de El Niño na região de Rio Largo, foi reduzida, ou seja, esses anos apresentaram maior tendência para ocorrências de períodos secos. Após esse período de eventos fortes de El Niño, notou-se diminuição nas probabilidades de ocorrências de períodos secos, relacionada à ocorrência da fase fria do ENOS (La Niña) entre 1983 a 1985. Em anos de La Niña houve aumento médio de precipitação pluvial, e consequentemente, poucos períodos secos (Souza et al., 2004).

A constatação dessa relação entre períodos secos e eventos de El Niño - Oscilação Sul, ainda foi acentuada com a 
ocorrência de um evento moderado de El Niño (1986 a 1988), onde as frequências de períodos secos voltaram a ser elevadas. O período no qual houveram as maiores probabilidades de ocorrência de períodos secos (1997 - 1998), coincidiu com um evento de El Niño forte. Esse período foi antecipado por um evento fraco de La Niña (1995 - 1996), e diminuição de períodos secos. Em todos os anos de El Niño forte e moderado (1983, 1987, 1991, 1993, 1997) foi observada maior frequência de períodos secos. Em anos de La Niña forte (1974, 1976, 1988), moderada (2000) e fraca (1996), a probabilidade de acontecer períodos secos diminui. Apesar de o fenômeno El Niño estar relacionado geralmente a eventos de secas no Nordeste do Brasil, sua ocorrência pode também não ter uma alta relação. Kane
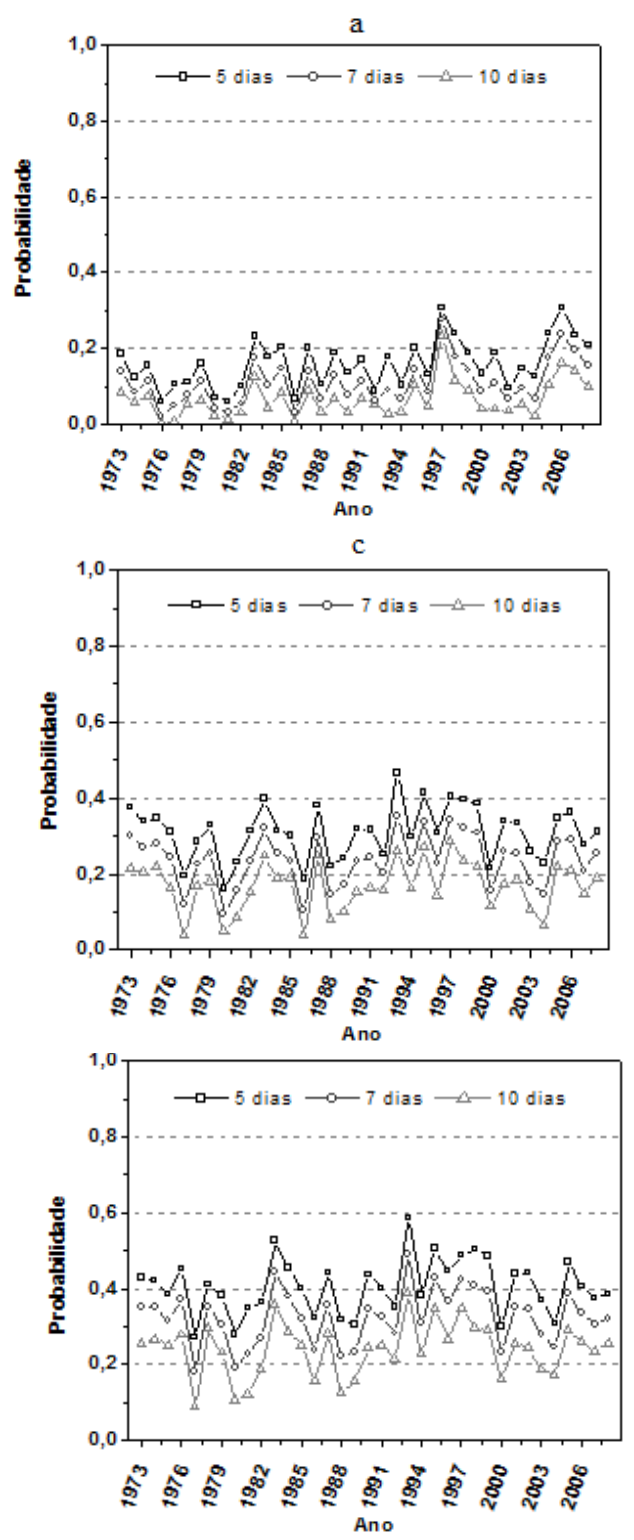

(2001) constatou que em aproximadamente $40 \%$ dos anos, o El Niño não apresentou relação com a ocorrência de secas, devido a influência da temperatura da superfície do Oceano Atlântico, que contribuem para seca ou excesso de chuvas no Nordeste. Porém, um evento de El Niño forte sempre esteve associada a secas de moderadas a severas (Molion e Bernardo, 2002).

Entre os anos de 1973 e 1980 houve redução significativa na probabilidade de ocorrência de períodos secos. Isso pode está associado a outro modo de variabilidade climática que é a Oscilação Decadal do Pacífico (ODP). Na fase fria da ODP, que ocorreu entre 1942 e 1976, houve maior incidência de eventos de La Niña, o que proporcionou a redução na probabilidade de ocorrência de períodos secos. Entre 1977 e 1998 ocorreu a fase
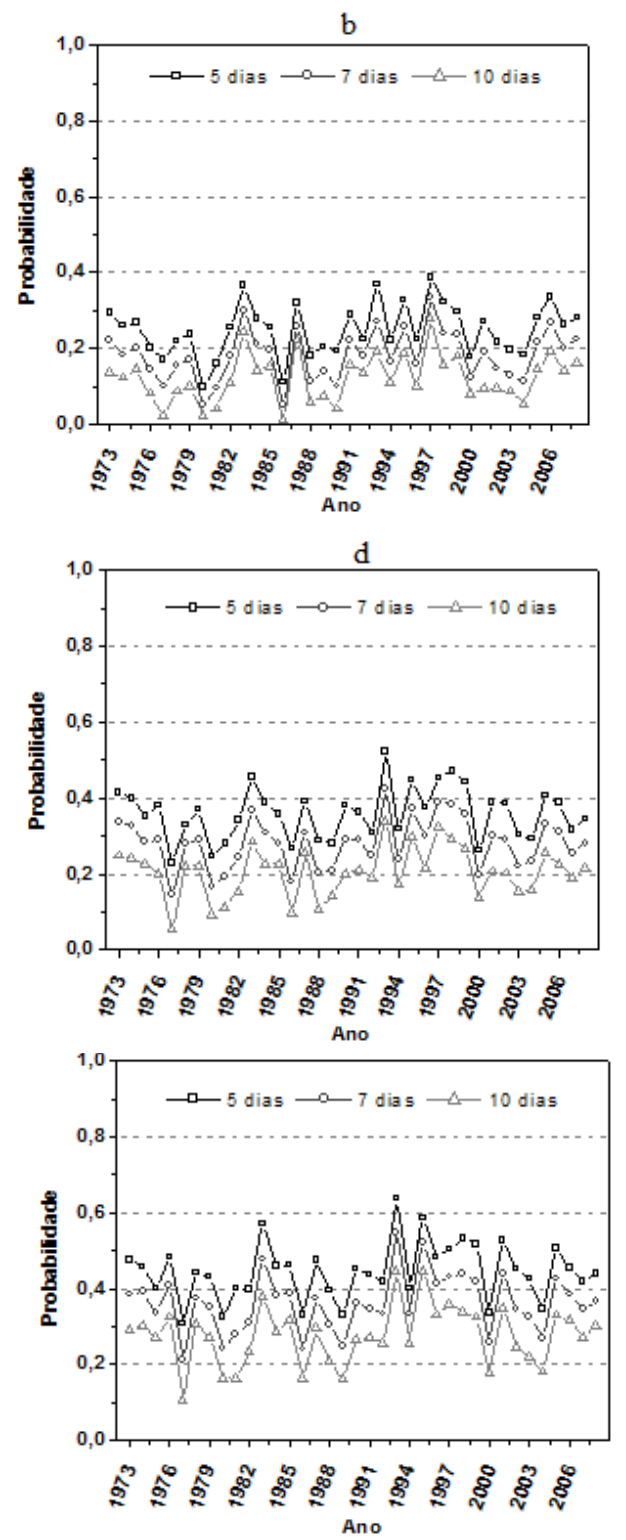

Figura 2 - Probabilidade média anual da ocorrência de períodos secos ( $\geq 5$ dias, $\geq 7$ dias, $\geq 10$ dias) no período de 1973 - 2008, Rio Largo - AL, considerando dias sem precipitação (a), $1 \mathrm{~mm}$ (b), $2 \mathrm{~mm}$ (c), $3 \mathrm{~mm}$ (d), $4 \mathrm{~mm}$ (e) e $5 \mathrm{~mm}$ (f), como limites de precipitação pluvial de dias secos. 
quente da ODP, que apresentou maior incidência de eventos El Niño. Nesse período foi registrado aumento das probabilidades de ocorrência de períodos secos.

\section{CONCLUSÕES}

As ocorrências de períodos secos apresentam grande variabilidade entre as estações seca e chuvosa. O período seco de 5 dias foi o mais frequente ( 5 a $85 \%$ ) durante os anos da série de estudo. Na estação chuvosa, os períodos secos maiores que 7 dias ( 0 a 45\%) e 10 dias ( 0 a 5\%) apresentam baixas ocorrências. Nota-se forte influência de eventos de ENOS (El Niño Oscilação Sul) na frequência dos períodos secos da região. Esses eventos provocam mudanças significativas nas ocorrências de períodos secos, sendo que anos com episódios de El Niño intensificam a frequência de períodos secos (10 a 60\%), enquanto eventos de La Niña reduzem essas frequência ( 0 a 45\%).

\section{AGRADECIMENTOS}

A Fapeal (Fundação de Amparo à Pesquisa do Estado de Alagoas), pelo financiamento deste trabalho através da concessão de bolsa de Mestrado. E as instituições ligadas aos seguintes projetos aprovados: FAPEAL, CT-HIDRO/CNPq504068/03-2, CNPq UNIVERSAL 479143/2007-2, CAPES, CNPq-PIBIC-UFAL.

\section{REFERÊNCIAS BIBLIOGRÁFICAS}

ANDRADE, A. R. S.; PAIXÃO, F. J. R.; AZEVEDO, C. A. V.; GOUVEIA, J. P. G.; OLIVEIRA JÚNIOR, J. A. S. Estudo do comportamento de períodos secos e chuvosos no município de Garanhuns, PE, para fins de planejamento agrícola. Revista Brasileira de Tecnologia Aplicada nas Ciências Agrárias, v.1, n.1, p.55-61, 2008.

ANDRADE JÚNIOR, A. S.; FRIZZONE, J. A.; SENTELHAS, P. C. Simulação da precipitação diária para Parnaíba e Teresina, PI, em planilha eletrônica. Revista Brasileira de Engenharia Agrícola e Ambiental, Campina Grande, v. 5, n. 2, p. 271-278, 2001.

ARRUDA, H. V. de; PINTO, H. S. An alternative model for dry-spell probability analysis. Monthly Weather Review, Boston, USA, v. 108, n. 6, p. 823-825, 1980.

ASSAD, E. D.; MASUTOMO, R.; SANO, E. E.; CASTRO, L. H.; SILVA, F. M. Veranico na região dos cerrados brasileiros: Freqüência e probabilidade de ocorrência. Pesquisa Agropecuária Brasileira, Brasília, v. 28, n. 9, p. 993-1003, 1993.

AZEVEDO, P. V.; LEITÃO, M. M. V. B. R. Aplicação de processos em cadeia de Markov às precipitações diárias no
Estado da Paraíba. Revista Brasileira de Meteorologia, v.5, n.1, 389-402, 1990.

BARRON, J.; ROCKSTROM, J.; GICHUKI, F.; HATIBU, N. Dry spell analysis and maize yields for two semiarid locations in East Africa. Agricultural and forest Meteorology, v. 117, p. 23 - 37, 2003.

BUISHAND, T. A. Stochastic Modelling of Daily Rainfall Sequences. Wageningen, Veenman and Zonen. p. 211. 1977. CARDIM, A. H. Caracterização da estação de cultivo em Alagoas: análise temporal e espacial. Maceió, 2003. 120 f. Dissertação(Mestrado em Meteorologia)-Instituto de Ciências Atmosféricas, Universidade Federal de Alagoas, 2003.

CARVAlHO, A. L. Estação de Cultivo Baseada na Precipitação Pluvial Diária e na Ocorrência de Períodos Secos para a Região de Rio Largo, Alagoas. Maceió, 2010. 76 f. Dissertação (Mestrado em Meteorologia) - Instituto de Ciências Atmosféricas, UniversidadeFederal deAlagoas, 2010.

FARMER, G.; WIGLEY, T. M. L. Climatic Trends for Tropical Africa: a research report of the Overseas Development Administration. Climatic Research Unit, University of East Anglia, p. 136. 1985.

FIETZ, C. R.; FRIZZONE, F. A.; FOLEGATTI, M. V. Probabilidade de ocorrência de períodos secos e chuvosos na região de Dourados, MS. Irriga, Botucatu, v.3, n.1, p. 16-22, 1998.

KANE, R. P. Limited effectiveness of El Niños in causing droughts in NE Brazil and the prominent role of Atlantic parameters. Revista Brasileira de Geofísica, Brasil, v. 19, n. 2, p. 231-236, 2001.

KELLER FILHO, T.; ZULLO JUNIOR, J.; LIMA, P. R. S. R. Análise da transição entre dias secos e chuvosos por meio da Cadeia de Markov de terceira ordem. Pesquisa Agropecuária Brasileira, v. 41, n. 9, p. 1341-1349, 2006. KOUSKY, V. E. Frontal influences on Northeast Brazil. Monthly Weather Review, Washington, v. 107, n. 9, p. 1140-1153, 1979.

KOUSKY, V. E. Diurnal raifall variation on Northeast Brazil. Monthly Weather Review, Washington, v. 108, n. 4, p. 488-498, 1980.

MARENGO, J. A.; SCHAEFFER, R.; ZEE, D.; PINTO, H. S. Mudanças climáticas e eventos extremos no Brasil. Disponível em: http://www.fbds.org.br/cop15/FBDS_ MudancasClimaticas.pdf. Acesso em Outubro de 2010.

MORSE, B. Foreword. Drought and Hunger in Africa. M. Glantz, Ed., Cambridge University Press, xiii-xx. 1987.

MOLION, L. C. B.; BERNARDO, S. Uma revisão da dinâmica das chuvas no Nordeste brasileiro. Revista Brasileira de Meteorologia, v.17, p.1-10, 2002.

NIEUWOLT, S. Estimating the agricultural risks of tropical rainfall. Agricultural and Forest Meteorology, v. 45, p. 251-263, 1989. 
PEREIRA, E. R. R.; FREITAS, J. C.; ANDRADE, A. R. S.; MACEDO, M. L. A.; SILVA NETO, M. F. Variabilidade do número de dias de chuva no estado de Alagoas. Revista Brasileira de Tecnologia Aplicada nas Ciências Agrárias, Guarapuava-PR, v.5, n.1, p.7-26, 2012.

SANTOS, C. A. C.; BRITO, J. I. B.; RAMANA RAO, T. V.; MENEZES, H. E. A. Tendências dos índices de precipitação no Estado do Ceará. Revista Brasileira de Meteorologia (Impresso), v. 24, p. 39-47, 2009.

SIVAKUMAR, M. V. K. Empirical analysis of dry drought spells for agricultural applications in West Africa. Journal of Climate. V. 5, p. 532-539. 1992.

SOUZA, J. L.; MOURA FILHO, G.; LYRA, R. F. F; TEODORO, I.; SANTOS, E. A.; SILVA, J. L.; SILVA, P. R. T.; CARDIM, A. H.; AMORIN, E. C. Análise da Precipitação Pluvial e Temperatura do Ar na Região do Tabuleiro Costeiro de Maceió, AL, Período 1972 - 2001. Revista Brasileira de Agrometeorologia, v.12, n.1, p.131 - 141, 2004.
STERN, R. D.; DENNETT, M. D.; DALE, I. C. Analysing rainfall measurements to give agronomically useful results. I. Direct Methods. Experimental Agriculture, v. 18, p. 223-236. 1982a.

STERN, R. D.; DENNETT, M. D.; DALE, I. C. Analysing rainfall measurements to give agronomically useful results. II. Modelling approach. Experimental Agriculture, v.18, p.237-253. 1982b.

STERN, R. D.; RIJKS, D.; DALE, I.; KNOCK, J. Instat Climatic Guide. p.325. Oct.2005.

VASCONCELLOS, S. L. B.; ANDRÉ, R. G. B.; PERECIN, D. Probabilidade de ocorrência de dias secos para a região de Jaboticabal-SP. Revista Brasileira de Agrometeorologia, Santa Maria, v. 11, n. 2, p. 321-325, 2003.

VIANA, T. V. A.; AZEVEDO, B. M.; BOMFIM, G. V.; ANDRADE JUNIOR, A. S. Probabilidade de ocorrência de períodos secos e chuvosos, em Pentecoste, CE. Irriga (Botucatu), Botucatu, v. 7, n. 03, p. 226-229, 2002. 